\title{
YAP1 enhances NF-KB-dependent and independent effects on clock-mediated unfolded protein responses and autophagy in sarcoma
}

\author{
Adrian Rivera-Reyes ${ }^{1}$, Shuai Ye ${ }^{1}$, Gloria E. Marino', Shaun Egolf', Gabrielle E. Ciotti', Susan Chor ${ }^{1}$, Ying Liu', $^{1}$ \\ Jessica M. Posimo ${ }^{1,2}$, Paul M. C. Park ${ }^{3}$, Koreana Pak , Yael Babichev" ${ }^{4,5}$, Jaimarie Sostre-Colón ${ }^{1}$, Feven Tameire ${ }^{6}$, \\ Nektaria Maria Leli ${ }^{6}$, Constantinos Koumenis ${ }^{6}$, Donita C. Brady (10,2, Anthony Mancuso ${ }^{7}$, Kristy Weber ${ }^{8}$, \\ Rebecca Gladdy, ${ }^{4,}$ Jun $\mathrm{Qi}^{3}$ and T. S. Karin Eisinger-Mathason ${ }^{1}$
}

\begin{abstract}
Terminal differentiation opposes proliferation in the vast majority of tissue types. As a result, loss of lineage differentiation is a hallmark of aggressive cancers, including soft tissue sarcomas (STS). Consistent with these observations, undifferentiated pleomorphic sarcoma (UPS), an STS subtype devoid of lineage markers, is among the most lethal sarcomas in adults. Though tissue-specific features are lost in these mesenchymal tumors they are most commonly diagnosed in skeletal muscle, and are thought to develop from transformed muscle progenitor cells. We have found that a combination of HDAC (Vorinostat) and BET bromodomain (JQ1) inhibition partially restores differentiation to skeletal muscle UPS cells and tissues, enforcing a myoblast-like identity. Importantly, differentiation is partially contingent upon downregulation of the Hippo pathway transcriptional effector Yes-associated protein 1 (YAP1) and nuclear factor (NF)-KB. Previously, we observed that Vorinostat/JQ1 inactivates YAP1 and restores oscillation of NF-KB in differentiating myoblasts. These effects correlate with reduced tumorigenesis, and enhanced differentiation. However, the mechanisms by which the Hippo/NF-kB axis impact differentiation remained unknown. Here, we report that YAP1 and NF-KB activity suppress circadian clock function, inhibiting differentiation and promoting proliferation. In most tissues, clock activation is antagonized by the unfolded protein response (UPR). However, skeletal muscle differentiation requires both Clock and UPR activity, suggesting the molecular link between them is unique in muscle. In skeletal muscle-derived UPS, we observed that YAP1 suppresses PERK and ATF6-mediated UPR target expression as well as clock genes. These pathways govern metabolic processes, including autophagy, and their disruption shifts metabolism toward cancer cell-associated glycolysis and hyper-proliferation. Treatment with Vorinostat/JQ1 inhibited glycolysis/MTOR signaling, activated the clock, and upregulated the UPR and autophagy via inhibition of YAP1/NF-KB. These findings support the use of epigenetic modulators to treat human UPS. In addition, we identify specific autophagy, UPR, and muscle differentiation-associated genes as potential biomarkers of treatment efficacy and differentiation.
\end{abstract}

\footnotetext{
Correspondence: T. S. Karin Eisinger-Mathason (karineis@pennmedicine.upenn. edu)

${ }^{1}$ Abramson Family Cancer Research Institute, Department of Pathology \& Laboratory Medicine, Penn Sarcoma Program, University of Pennsylvania Perelman School of Medicine, Philadelphia, PA, USA

${ }^{2}$ Department of Cancer Biology, University of Pennsylvania Perelman School of Medicine, Philadelphia, PA, USA

Full list of author information is available at the end of the article These authors contributed equally: Adrian Rivera-Reyes and Shuai Ye Edited by R Aqeilan
}

\section{Introduction}

Soft tissue sarcomas (STS) are a complex set of tumors that arise in mesenchymal tissues, including muscle, fat, cartilage, and connective tissue. Owing to their karyotype complexity, variety of subtypes, and the lack of known drivers, adult sarcomas are very poorly understood.

\section{(c) The Author(s) 2018}

(c) (i) Open Access This article is licensed under a Creative Commons Attribution 4.0 International License, which permits use, sharing, adaptation, distribution and reproduction cc in any medium or format, as long as you give appropriate credit to the original author(s) and the source, provide a link to the Creative Commons license, and indicate if changes were made. The images or other third party material in this article are included in the article's Creative Commons license, unless indicated otherwise in a credit line to the material. If material is not included in the article's Creative Commons license and your intended use is not permitted by statutory regulation or exceeds the permitted use, you will need to obtain permission directly from the copyright holder. To view a copy of this license, visit http://creativecommons.org/licenses/by/4.0/. 
Treatment options are generally limited to radiation and surgery, as inadequate characterization has precluded the development of targeted therapies ${ }^{1-3}$. Our current work focuses on undifferentiated pleomorphic sarcoma (UPS), an aggressive adult tumor found in skeletal muscle. Muscle-derived UPS is a commonly diagnosed subtype relative to other sarcomas and is particularly difficult to treat ${ }^{4}$. We found that the central Hippo effector, Yesassociated protein 1 (YAP1), is stabilized in human UPS tumors and promotes a pro-proliferation transcriptional program $^{5,6}$. YAP1 is unusually stable in UPS and potentially other sarcomas due to epigenetic silencing of its inhibitor, Angiomotin (AMOT) ${ }^{7}$, and Hippo kinase copy number loss ${ }^{5}$. These perturbations stabilize YAP1 at the protein level; enhance its nuclear localization and subsequent transcriptional activity ${ }^{8}$. Though well-studied in epithelial tumors, the specific downstream effectors of YAP1 in sarcomas are not well characterized.

Skeletal muscle-derived UPS is thought to develop from muscle progenitor cells/satellite cells ${ }^{9}$, which undergo proliferation as immature myoblasts before differentiating into mature muscle fibers. YAP1 and NF- $\mathrm{kB}$ signaling are essential for myoblast proliferation and these pathways must be inhibited to permit terminal differentiation ${ }^{10-14}$. Thus, during normal muscle development inhibition of $\mathrm{NF}-\mathrm{kB}$ and YAP1 are associated with loss of proliferative capacity, and upregulation of muscle differentiation markers like MYOD and MEF2C. Recently, we discovered that YAP1 controls NF- $\mathrm{KB}$ activity in muscle-derived UPS, by inhibiting expression of ubiquitin specific peptidase 31 (USP31) a negative regulator of NF- $\kappa B^{7}$. In the absence of a specific inhibitor for YAP1 we used a combination of the epigenetic modulators suberoylanilide hyroxamic acid (SAHA; Vorinostat), and the BET bromodomain inhibitor JQ1, which we recently discovered suppresses YAP1 activity. Though SAHA/JQ1 treatment has widespread effects, we use these tools to interrogate and then validate YAP1-mediated signaling and phenotypes. Importantly, SAHA/JQ1 treatment upregulated a transcriptional program associated with muscle differentiation in UPS cells. Here we report that inhibition of YAP1 and/or NF-kB recapitulates several key aspects of SAHA/JQ1-mediated differentiation.

Interestingly, we observed that NF- $\mathrm{kB}$ signaling oscillates over time in muscle precursor cells ${ }^{7}$ and other tissues $^{15,16}$. Consistent with these findings, normal myoblast proliferation and muscle differentiation have been linked to peripheral circadian oscillation ${ }^{17-19}$. The circadian clock is a 24-hour molecular signaling hub that regulates proliferation via control of metabolic processes ${ }^{20,21}$ and is regulated by positive and negative feedback loops ${ }^{22,23}$. The main transcriptional components, CLOCK and BMAL1, form a heterodimer that binds to an E-box in the promoters of target genes, such as PERIOD (PER) and
CRYPTOCHROME (CRY), enhancing their expression, which in turn inactivates CLOCK and BMAL1 heterodimers $^{23,24}$. Shuttling of PER and CRY proteins from the cytoplasm to the nucleus regulates the feedback loop. Importantly, a growing body of literature suggests that disruption of circadian oscillation promotes tumorigenesis in a variety of cancer settings ${ }^{25-28}$.

Recent studies have shown that clock activity is linked to a number of pathways associated with muscle differentiation including the unfolded protein response $(\mathrm{UPR})^{29-31}$ and autophagy ${ }^{21,32-34}$. Cross-talk between UPR and autophagy has also been observed but the molecular mechanisms and coordination of these processes have yet to be elucidated ${ }^{35-38}$. Importantly, YAP1 plays a role in cancer related autophagic flux ${ }^{39}$ and $\mathrm{UPR}^{40}$. Based on these observations we hypothesized that YAP1 and/or NF- $\mathrm{kB}$ helps control differentiation in muscle tissues and tumors via regulation of the circadian clock and downstream processes. Here we show that YAP1/NF$\kappa \mathrm{B}$, control the switch between differentiation and proliferation in sarcoma by suppressing the circadian clock and UPR. Interestingly, we observed that YAP1 also suppresses autophagy but in an NF-kB-independent manner.

\section{Materials and methods \\ Mouse models \\ Genetically Engineered Mouse Models (GEMM)}

All experiments were performed in accordance with NIH guidelines and were approved by the University of Pennsylvania Institutional Animal Care and Use Committee. We generated $\mathrm{Kras}^{\mathrm{G12D+}}$; Trp53 $3^{\mathrm{fl} / f l} ; \mathrm{YAP}^{\mathrm{fl} / \mathrm{fl}}$ (KPY) and Kras ${ }^{G 12 D+}$; Trp53 $3^{f l f l}$; Rela ${ }^{f l f f l}$ (KPR) mice by crossing KP with YAP1 $1^{f l f l}$ and Rela ${ }^{f l f l}$ animals. Tumors were generated by injection of a calcium phosphate precipitate of adenovirus expressing Cre recombinase (University of Iowa) into the right gastrocnemius muscle of 3-6-month-old mice.

\section{In vivo drug treatment}

For in vivo drug studies, total $44(n=11$ per group) autochthonous KP mice were randomly divided into four groups to receive different treatments once tumors reached $100 \mathrm{~mm}^{3}$, and injected for up to 20 days. The mice are killed $24 \mathrm{~h}$ after the tumor volume reaches 2000 $\left.\mathrm{mm}^{3}\right)$. (1) Vehicle group (10\% Hydroxypropyl- $\beta$ cyclodextrin plus dimethyl sulfoxide (DMSO) was diluted daily in sterile $45 \% \mathrm{PEG} / 55 \% \mathrm{H}_{2} \mathrm{O}$ ); (2) JQ1 and SAHA combination treatment group (drug were diluted in respective vehicles). Treatment method for drug combination group: (1) $25 \mathrm{mg} / \mathrm{kg}$ SAHA $+50 \mathrm{mg} / \mathrm{kg}$ JQ1 for first 5 days. (2) $25 \mathrm{mg} / \mathrm{kg}$ SAHA $+25 \mathrm{mg} / \mathrm{kg} \mathrm{JQ1}$ each other day for 10 days. (3) $25 \mathrm{mg} / \mathrm{kg}$ SAHA $+50 \mathrm{mg} / \mathrm{kg}$ JQ1 for 2 days. (4) Then mice with tumors received 25 
$\mathrm{mg} / \mathrm{kg}$ SAHA with $25 \mathrm{mg} / \mathrm{kg}$ JQ1 for 3 days. Mice without tumors received $5 \mathrm{mg} / \mathrm{kg}$ SAHA and $5 \mathrm{mg} / \mathrm{kg}$ JQ1 for 3 days). JQ1 was provided by Jun Qi (Dana-Farber Cancer Institute) and SAHA was purchased from Cayman Chemical. HP- $\beta-C D$ and PEG400 were obtained from SigmaAldrich.

\section{Oncomine and TCGA survival analysis}

We used the publically available database Detwiller et al. via the Oncomine Research Premium edition software (version 4.5, life Technologies) to query PER1, PER2, CRY1, CRY2, ARNTL, TXNIP, DDIT3, FASN, CPT1A, $C P T 1 B$ gene expression in MFH/UPS. We also evaluated human patient survival using the TCGA sarcoma data set. Kaplan-Meier analyses were performed for overall survival of patients.

\section{Cell lines}

KP230, KP250, and KIA cell lines were derived from UPS mouse tumors as described $\mathrm{in}^{41}$. Human HT-1080, HEK-293T cell lines were purchased from ATCC (Manassas, VA, USA). STS-109 cell line was derived from human UPS patients. STR analysis was performed at the time of derivation and confirmed in April 2015. Cells were purchased, thawed, and then expanded in the laboratory. Multiple aliquots were frozen down within 10 days of initial resuscitation. For experimental use, aliquots were resuscitated and cultured for up to 20 passages (4-6 weeks) before being discarded. Cells were cultured in Dulbecco's Modified Eagle's medium (DMEM) with 10\% (vol/vol) fetal bovine serum (FBS), 1\% penicillin/streptomycin, $1 \%$ glutamine, at $5 \% \mathrm{CO}_{2}$ and $37^{\circ} \mathrm{C}$. All cell lines were confirmed to be negative for mycoplasma contamination.

\section{Drug treatments}

Cells were treated with SAHA $(2 \mu \mathrm{M})$ and JQ1 $(0.5 \mu \mathrm{M})$ either individually or in combination for the time indicated in the figure legends. Drugs were refreshed for any cells treated for longer than $48 \mathrm{~h}$.

\section{Lentiviral transduction}

shRNA-mediated TRCN0000075403; Arntl: TRCN0000095055; Txnip: TRCN0000182360; Ddit3: TRCN0000103709; RelA: TRCN0000055344, TRCN0000055346; and Yap1: TRCN0000095864, TRCN0000095865 were obtained as glycerol stocks from Dharmacon. Scramble shRNA was obtained from Addgene. High-copy plasmid purification was conducted for each shRNA using Clontech Laboratories Inc. NucleoBond Exta Midi kit (740410.50) according to the NucleoBond ${ }^{\circledR}$ Xtra Plasmid Purification Maxi protocol. shRNA plasmids were packaged by using the third-generation lenti-vector system (VSV-G, p-
MDLG, and pRSV-REV) and expressed in HEK-293T cells. Supernatant was collected at 24 and $48 \mathrm{~h}$ after transfection and subsequently concentrated by using 10kDa Amicon Ultra-15 centrifugal filter units (Millipore). After $72 \mathrm{~h}$ of lentiviral infection, cells were selected with puromycin $(1.5 \mu \mathrm{g} / \mathrm{ml})$ for $24-48 \mathrm{~h}$. shRNA infected cells were treated with SAHA $(2 \mu \mathrm{M})$ and JQ1 $(0.5 \mu \mathrm{M})$ for $48 \mathrm{~h}$ kept under puromycin-selecting conditions.

\section{ChIP-seq}

For tumor samples resected from UPS patients at the Hospital of the University of Pennsylvania, 100 mg of tissue was minced into $1-2 \mathrm{~mm}$ pieces and incubated in $1 \%$ formaldehyde for $15 \mathrm{~min}$. Formaldehyde was quenched with glycine at $0.125 \mathrm{M}$. Fixed tissue was homogenized for $60 \mathrm{sec}$ with a Tissue Tearor Homogenizer (Biospec) at 30,000 rounds per minute. Homogenized tissue was washed with ice-cold phosphate-buffered saline (PBS) with $1 \times$ HALT protease inhibitor. For cell line ChIP-RX, samples were fixed for $10 \mathrm{~min}$ in $1 \%$ formaldehyde quenched with glycine and washed with PBS as above. 5e6 S2 cells (Drosophila Melanogaster) were added to each sample of 2.5e7 for ChIP-RX normalization in downstream analysis.

\section{Transient transfections}

SMARTpool: ON-TARGETplus Yap1 siRNA (M100439-01-0005), Cry2 siRNA (L-040486-00-0005), Per1 siRNA (L-040487-00-0005), and non-targeting siRNA were purchased from Dharmacon.

\section{UPR reporter assays}

KP cells were plated in a six-well plate and transiently transfected using the Lipofectamine ${ }^{\mathrm{TM}} 3000$ protocol. Cells were transfected using the Addgene plasmids ATF4 5: 5'ATF4:GFP (\#21852) and pEGFP-ATF6 (\#32955). Fluorescence images were taken using Olympus IX2-UCB microscope, SensiCam ${ }^{\mathrm{QE}}$ High Perfomance camera, and $\mathrm{X}$-Cite Series 120PC. Images were taken using the Slidebook 6 program.

\section{Immunoblots}

Protein lysate was prepared in SDS/Tris (pH7.6) lysis buffer, separated by electrophoresis in $8-10 \%$ sodium dodecyl sulphate-polyacrylamide gel electrophoresis gels, transferred to nitrocellulose membrane, blocked in 5\% non-fat dry milk, and probed with the following antibodies: rabbit anti-PER1 (ab3443; 1:250), rabbit antiCRY2 (ab38872, 1:500) (Abcam), rabbit anti-BMAL1 (14020S; 1:1000), rabbit anti-YAP1 (4912; 1:1000), rabbit anti-GAPDH (2118; 1:1000), (Cell Signaling Technology), rabbit anti-CPT1A (15184-1-AP; 1:1000) (Proteintech), rabbi anti-TXNIP (ab188865, 1:1000) (Abcam), rabbit anti-CHOP (60304-1, Ig, 1:1000) (Proteintech), rabbit 
anti-FASN (3081, 1:1000) (Cell Signaling Technology), anti-LC3A/B (12741, 1:1000) (Cell Signaling Technology), rabbit anti-Caspase-3 (9662; 1:1000).

\section{qRT-PCR}

Total RNA was isolated from tissues and cells using the TRIzol regent (Life Technologies) and RNeasy Mini Kit (Qiagen). Reverse transcription of mRNA was performed using the High-Capacity RNA-to-cDNA Kit (Life Technologies). qRT-PCR was performed by using a ViiA7 apparatus. All probes were obtained from TaqMan "best coverage" (Life Technologies). Hypoxanthine phosphoribosyltransferase and or/succinate dehydrogenase subunit A (SDHA) was used as an endogenous control.

\section{Luciferase assay}

Plasmid pABpuro-BluF (46824; Addgene) was transfected into 293T cells (ATCC) to generate lentiviral particles in the supernatant. Viral supernatant was harvested and then concentrated by centrifugal filter units (Amicon Ultra-15, Millipore). Then Bmal reporter virus was transduced into KP230 cells. Positive Bmall reporter cells were selected by puromycin. For shRNA assays the Bmal reporter cell line was transduced with lentivirus expressing control or Yap1 shRNA. For drug studies, the Bmal reporter cells were treated with SAHA $(2 \mu \mathrm{M}) / \mathrm{JQ} 1(0.5$ $\mu \mathrm{M})$ on time course. Luciferase activity was assayed using the Dual Luciferase Assay System (E2920, Promega) according to the manufacturer's protocol on a Luminometer (GLOMAX, Promega). Results were calculated as fold induction.

\section{Microarray and gene set enrichment analysis}

Microarray services were provided by the UPENN Molecular Profiling Facility, including quality control tests of the total RNA samples by Agilent Bioanalyzer and Nanodrop spectrophotometry. All protocols were conducted as described in the Affymetrix WT Plus Reagent Kit Manual and the Affymetrix GeneChip Expression Analysis Technical Manual. In brief, $250 \mathrm{ng}$ of total RNA was converted to first-strand cDNA using reverse transcriptase primed by poly $(\mathrm{T})$ and random oligomers that incorporated the T7 promoter sequence. Second-strand cDNA synthesis was followed by in vitro transcription with T7 RNA polymerase for linear amplification of each transcript, and the resulting complementary RNA was converted to complementary DNA (cDNA), fragmented, assessed by Bioanalyzer, and biotinylated by terminal transferase end labeling. Five and a half micrograms of labeled cDNA were added to Affymetrix hybridization cocktails, heated at $99^{\circ} \mathrm{C}$ for $5 \mathrm{~min}$ and hybridized for $16 \mathrm{~h}$ at $45^{\circ} \mathrm{C}$ to Mouse Transcriptome $1.0 \mathrm{ST}$ GeneChips (Affymetrix Inc., Santa Clara CA) using the GeneChip Hybridization oven 645 . The microarrays were then washed at low $(6 \times$ sodium chloride sodium phosphateEDTA buffer) and high (100mM MES, 0.1M NaCl) stringency and stained with streptavidin-phycoerythrin. Fluorescence was amplified by adding biotinylated antistreptavidin and an additional aliquot of streptavidin-phycoerythrin stain. A GeneChip 3000 7G scanner was used to collect fluorescence signal. Affymetrix Command Console and Expression Console were used to quantitate expression levels for targeted genes; default values provided by Affymetrix were applied to all analysis parameters. Affymetrix cel (probe intensity) files were normalized and summarized using RMA-SST to the gene level using Expression Console software (v1.4.1). Inter sample variation was visualized using Principal Components Analysis in Partek Genomics Suite (v6.6, Partek, Inc., St. Louis, MO). Differential gene expression was tested using Significance Analysis of Microarrays (samr v2.0), yielding fold change, $q$ value (false discovery rate) and d-score for each gene. We observed a small number of genes meeting our cutoffs for differential expression and so proceeded to gene set enrichment analysis (GSEA). $\log _{2}$-transformed RMA-sst expression values were used as input to GSEA $^{42}$ where enrichment was tested against the hallmark gene sets from the Molecular Signatures Database (MSigDB, v5.1, http:// software.broadinstitute.org/gsea/msigdb/index.jsp)

\section{Accession codes}

Sequencing data reported in this paper have been deposited in NCBI's Gene Expression Omnibus and are accessible through GEO Series accession numbers GSE109920 and GSE109923.

\section{C2C12 growth and differentiation}

C2C12 murine myoblast cells were obtained from ATCC (Manassas, VA, USA). The cells grow as undifferentiated myoblasts in growth medium (20\% FBS with $1 \%$ penicillin/streptomycin), and were passaged every $2-3$ days at $50 \%$ sub-confluence. To induce differentiation cells were grown overnight to $\sim 80 \%$ confluence in growth medium, and then switched to DMEM supplemented with $2 \%$ horse serum. Differentiation media was refreshed every 2 days.

\section{Immunohistochemistry}

Human UPS paraffin-embedded tissues were obtained from the Surgical Pathology group at University of Pennsylvania and stained thioredoxin-interacting protein (TXNIP) and C/EBP homologous protein (CHOP). Murine tumors from the KP GEMM and KP allografts were also sectioned and stained. IHC was performed on 5micron tissue sections according to standard protocols. Sections were deparaffinized, rehydrated, and subjected to epitope retrieval and stained with The following antibody 
concentrations were used: rabbit anti-TXNIP (ab188865; 1:100) (Abcam), mouse anti-CHOP (Proteintech) (603041-IG; 1:250), rabbit anti-LC3B (Novus Biologicals; NB1002220, 1:1000), rabbit anti-p62 (MBL International, PM045, 1:2000), mouse anti- Gadd34 (Novus Biologicals; NBP2-01787, 1:100) followed by peroxidase-based detection and counterstaining with haematoxylin using the Leica Bond $\mathrm{Rx}^{\mathrm{m}}$ system with conditions described previously ${ }^{43}$. Representative photographs were taken on a Leica DMI6000B inverted light and fluorescent microscope with a $40 \times$ oil objective. Images were blinded and positive staining was assessed in Image J. The color deconvolution macro was applied to images resulting in a 3,3'-diaminobenzidine staining (Color_2) generated window $^{44}$. Using the threshold function, the total area of the tumor in pixels was recorded utilizing the same parameters for each tumor image. Areas staining positive by these parameters were selected and the positive-staining area in pixels was recorded. The positive-staining area of the tumor in pixels was divided by the total area of the tumor in pixels to determine the percentage positivestaining area.

\section{Gas chromatography/mass spectrometry}

After growth overnight, cultures were treated with either DMSO or $2 \mu \mathrm{m}$ SAHA/0.5 $\mu \mathrm{M}$ JQ1. The cultures were allowed to grow for $48 \mathrm{~h}$. Subsequently, the medium was changed to DMEM that contained $7 \mathrm{mM}$ glucose, 4 $\mathrm{mM}$ glutamine, $75 \mu \mathrm{M}\left[\mathrm{U}_{-}{ }^{13} \mathrm{C}_{16}\right]$ palmitic acid and $75 \mu \mathrm{M}$ $\left[\mathrm{U}_{-}{ }^{13} \mathrm{C}_{18}\right.$ ] oleic acid plus either DMSO or $\mathrm{S} / \mathrm{J}$. Both fatty acids were bound to fatty acid free albumin (two moles fatty acid per mole of albumin) before they were added to the medium. After $12 \mathrm{~h}$ of growth in the ${ }^{13} \mathrm{C}$-labeled medium, the cultures were harvested by cold methanol extraction. The cold methanol (80:20 methanol:water) was pre-cooled to $-80^{\circ} \mathrm{C}$ and added rapidly $(2 \mathrm{ml} /$ dish) after removal of the extracellular medium to prevent loss of intracellular metabolites. The cultures were stored at $80^{\circ} \mathrm{C}$ prior to gas chromatography/mass spectrometry analysis (GC/MS) analysis. The methanol/cell mixtures were sonicated for $60 \mathrm{sec}$ with a probe sonicator to disrupt all cell membranes and then centrifuged at $13,000 \times \mathrm{g}$ for $10 \mathrm{~min}$. The supernatants were removed and transferred to sealable 4-ml glass tubes. Methanol and water were removed from the cell extracts with a heated $\left(45^{\circ} \mathrm{C}\right)$ nitrogen evaporator. For GC/MS, the extracts were first derivatized with $\mathrm{N}, \mathrm{O}-\mathrm{Bis}$ (trimethylsilyl) trifluoroacetamide (BSTFA). The extracts in the 4-ml glass tubes were dissolved in $60 \mu \mathrm{l}$ of pyridine. Subsequently, $60 \mu \mathrm{l}$ BSTFA with 1\% trimethylchlorosilane (SigmaAldrich, St. Louis, MO) were added and the mixtures were heated to $55 \mathrm{C}$ for $60 \mathrm{~min}$. After cooling, the reaction mixtures were centrifuged at $13,000 \times \mathrm{g}$ for $5 \mathrm{~min}$. The supernatant was transferred to $1.5 \mathrm{ml}$ capped injection vials that were fitted with volume reducing glass sleeves. The derivatized samples were analyzed with an Agilent 7890A/5975C GC/MS system. Mass fragments were generated by electron impact at $70 \mathrm{eV}$. Helium was used as the carrier gas for the GC DB-5 column ( $30 \mathrm{~m}$, with 10 $\mathrm{m}$ empty pre-column) at a flow rate of $1 \mathrm{ml} / \mathrm{min}$. The injector was operated in splitless mode at $250^{\circ} \mathrm{C}$. The column temperature profile was $0-3 \mathrm{~min}: 100^{\circ} \mathrm{C}, 3-17$ min: $\operatorname{ramp} 10^{\circ} \mathrm{C} / \mathrm{min}, 17-47 \mathrm{~min}: 240^{\circ} \mathrm{C}, 47-52 \mathrm{~min}$ : $300^{\circ} \mathrm{C}$. The citrate retention time was $24.1 \mathrm{~min}$. The mass scan range was 50-550 daltons. The relative enrichment of metabolites was calculated using IsoCor ${ }^{45}$.

\section{Statistical analysis}

Statistical analysis was performed using Prism (Graph Pad Software). Data are shown as mean \pm standard error of mean or deviation. Data were reported as biological replicates. Experiments were performed in triplicate. Student $t$ tests (unpaired two tailed) were performed to determine whether a difference between two values is statistically significantly different, with a $P$ value $<0.05$ considered significant.

\section{Results}

Clock gene expression is high in differentiating muscle and lost in UPS

We investigated the role of YAP1 using the $L S L$ Kras $^{\mathrm{G} 12 \mathrm{D} /+} ; \operatorname{Tr}^{\mathrm{f}} 53^{\mathrm{fl} / \mathrm{fl}}$ (KP) model of UPS. Adenovirus expressing Cre recombinase injected into the gastrocnemius muscle activates oncogenic Kras expression and deletes p53 in muscle progenitor cells, resulting in tumors that recapitulate human UPS ${ }^{46,47}$. Though Kras mutation is rare in human sarcomas, hyperactivation of the MAPK pathway downstream of activated KRAS is common in UPS and is an excellent prognostic indicator ${ }^{48}$. Importantly, Yap1 is stabilized in KP tumors ${ }^{7}$.

We crossed $\operatorname{Yap}^{\mathrm{fl} / \mathrm{fl}}$ and KP mice to generate $L S L$ $\operatorname{Kras}^{\mathrm{G} 12 \mathrm{D} /+} ; \operatorname{Trp} 53^{\mathrm{fl} / \mathrm{fl}} ; \operatorname{Yap1}^{\mathrm{fl} / \mathrm{fl}}$ (KPY) animals and saw increased tumor latency and reduced tumor weight and volume, relative to $\mathrm{KP}^{7}$. To determine the functional role of Yap1 in UPS we performed microarray analysis of 5 $\mathrm{KP} / \mathrm{KPY}$ tumors and observed differential expression of circadian clock genes (Fig. 1a), validated by qRT-PCR of RNA isolated from murine tumors (Fig. 1b). Circadian clock function is important for skeletal muscle mass maintenance and function ${ }^{17,49}$. In differentiating $\mathrm{C} 2 \mathrm{C} 12$ murine myoblasts, we found increased expression of myogenesis-related targets (Fig. 1c), the Yap1 inhibitor Amot, clock genes, and the NF-kB-negative regulator, Usp31 (Fig. 1d). We also observed enhanced Per1 and Per2 protein expression, which correlated with Yap1 loss (Fig. 1e). Therefore, we hypothesized that loss of clock gene expression results in de-differentiation and sarcomagenesis. Consistently, PER1, PER2, and CRY2 are 


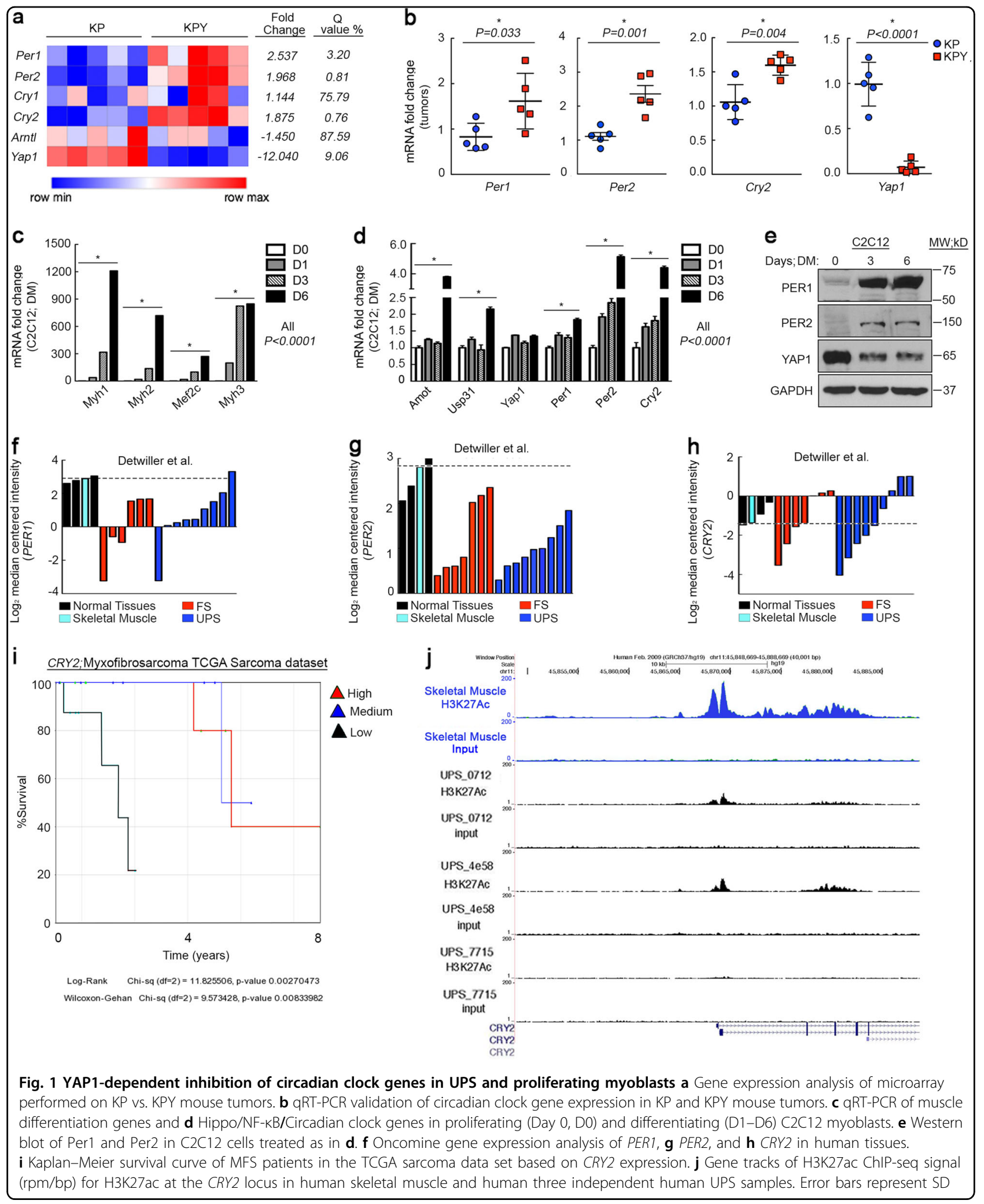


inhibited in human fibrosarcoma and UPS (Detwiller dataset) relative to normal skeletal muscle ${ }^{50}$ (Fig. 1f-h). Some fibrosarcomas, including myxofibrosarcomas, are now thought to be genetically indistinguishable from UPS $^{51}$ and are therefore included in our studies. We investigated the relationship between long-term survival and clock gene expression and found that low levels of $C R Y 2$ are associated with poor survival (Fig. 1i). To further assess CRY2 expression we performed RNA-seq and ChIP-seq, using Histone 3-lysine 27 Acetylation (H3K27Ac) antibody in human skeletal muscle and multiple human UPS samples. CRY2 expression is suppressed in UPS tumors compared with muscle ${ }^{7}$ and enrichment of H3K27Ac is lost in UPS at the CRY2 locus (Fig.1j). H3K27Ac enrichment is associated with active transcription and potential enhancer activity. The PER1 locus shows a similar pattern of H3K27Ac enrichment/gene expression (Supplementary Fig. S1a) but there was no statistically significant link between PER1 and patient survival likely due to the relatively low number of samples.

YAP1 suppresses circadian clock gene expression in UPS

We previously reported that YAP1 is downregulated in sarcoma cells treated with SAHA/JQ1 in vitro and in vivo and that ectopic expression of constitutively active YAP1 (YAPS6A) can rescue $\sim 40 \%$ of proliferation loss associated with treatment ${ }^{7}$. We hypothesized that SAHA/JQ1 might reactivate clock gene expression owing to loss of YAP1 and observed upregulation of clock genes and proteins in treated mouse and human UPS cells (Fig. 2a-e), and HT-1080 human fibrosarcoma cells (Supplementary Fig. S1b). The subset of YAP1-dependent clock genes varied between in vivo and in vitro assays. However, YAP1 inhibition (shRNA) phenocopied SAHA/ JQ1 treatment in KP cells, suggesting that any difference in target expression is due to experimental approach (Supplementary Fig. S1c). Lastly, PER1 and CRY2 antibodies were validated using siRNA (Supplementary Fig. S1d and e).

To determine whether YAP1 inhibition activates clock function we performed a Bmal luciferase reporter assay in KP cells using Yap1-specific siRNA, shRNA, and SAHA/ JQ1. We observed increased Bmal reporter expression in all three systems, indicating that Bmal/circadian clock activity is YAP1-dependent (Fig. 2f, g and Supplementary Fig. S2a). To verify that clock reactivation suppresses proliferation in Yap1-depleted cells we performed an in vitro rescue assay using Yap1- and Arntl-specific shRNAs. We observed a $\sim 40 \%$ proliferation rescue in double knockdown cells relative to cells expressing Yap1 shRNA alone (black bar vs. gray bar) (Fig. 2h, left), which correlated with a $\sim 40 \%$ reduction of Arntl expression in double knockdown cells relative to Yap1 shRNA alone (Fig. 2h, right and Supplementary
Fig. S2b) (black bar vs. gray bar). This partial rescue suggested that other mechanisms are at play. Given that YAP1 can have anti-apoptotic effects ${ }^{52,53}$, we hypothesized that YAP1 loss initiated apoptosis in a significant portion of cells and therefore proliferation could not be rescued by clock suppression. Loss of Yap1 resulted in cell cycle arrest (Supplementary Fig. S2c) and increased caspase-3 cleavage in KP cells, indicating enhanced apoptosis (Fig. 2i). Thus, although our rescue phenotype may appear modest we conclude that we are rescuing proliferation in a significant fraction of non-apoptotic cells.

\section{Circadian clock genes are regulated by NF-KB downstream of YAP1}

YAP1 enhances UPS proliferation in part via upregulation of persistent NF- $\mathrm{kB}$ signaling ${ }^{7}$. Here, we interrogated the role of NF- $\mathrm{kB}$ in UPS and clock reactivation. We bred Rela ${ }^{f l f l}$ mice into our KP model and found that genetic deletion of NF- $\mathrm{KB}$, encoded by the Rela gene, prevents outgrowth of tumors (Fig. 3a, b). Phosphorylation of the p65 subunit of NF- $\mathrm{kB}$ indicates its transcriptional activation. Both KP tumors and subcutaneous xenografts of human UPS cells stain positively for YAP1 and phospho-p65 (Fig. 3c). We investigated whether YAP1 repression of clock genes is mediated by NF- $\mathrm{kB}$ and

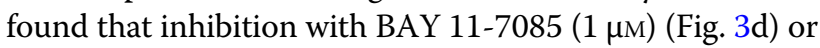
Rela shRNA increased clock gene expression (Fig. 3e). Importantly, NF- $\mathrm{kB}$ target expression oscillates over time in differentiating myoblasts ${ }^{7}$ and other cells ${ }^{15}$. YAP1 suppresses NF- $\mathrm{kB}$ oscillation, promoting persistently high activity, by controlling expression of USP $31^{7}$. Usp31 also oscillates in differentiating $\mathrm{C} 2 \mathrm{C} 12$ cells (Fig. 1d) as well as human STS-109 (Fig. 3f), KP, and HT1080 cells (Fig. 3g) treated with SAHA/JQ1 for $0-120 \mathrm{~h}$. Interestingly, the period of oscillation varies with the cellular proliferation rate. Slower proliferation rates (KP > HT-1080 > STS-109) correlate with shorter time to maximal USP31 induction by SAHA/JQ1. Ultimately Usp31 is lost in KPY tumors relative to KP (Supplementary Fig. S3a and S3b). This finding is consistent with our Bmal luciferase reporter assay in which we observe that SAHA/JQ1 treatment initially induces clock activity followed by inhibition (Fig. 2g). Our findings suggest the provocative hypothesis that NF-kB oscillation may drive the circadian cycle in muscle and muscle-derived sarcoma subtypes. To verify that clock reactivation suppresses proliferation in NF-kB-depleted cells we performed an in vitro rescue assay using Rela- and Arntl-specific shRNAs (Fig. 3h, i). We observed $\sim 20 \%$ rescue in proliferation (black bar vs. gray bar) (Fig. 3h, left), which correlates with reduction of Arntl in double knockdown cells (black bar vs. gray bar) (Fig. 3h, right). Similarly, BAY 11-7085 (1 $\mu \mathrm{M})$ dramatically decreased KP cell proliferation, which was 


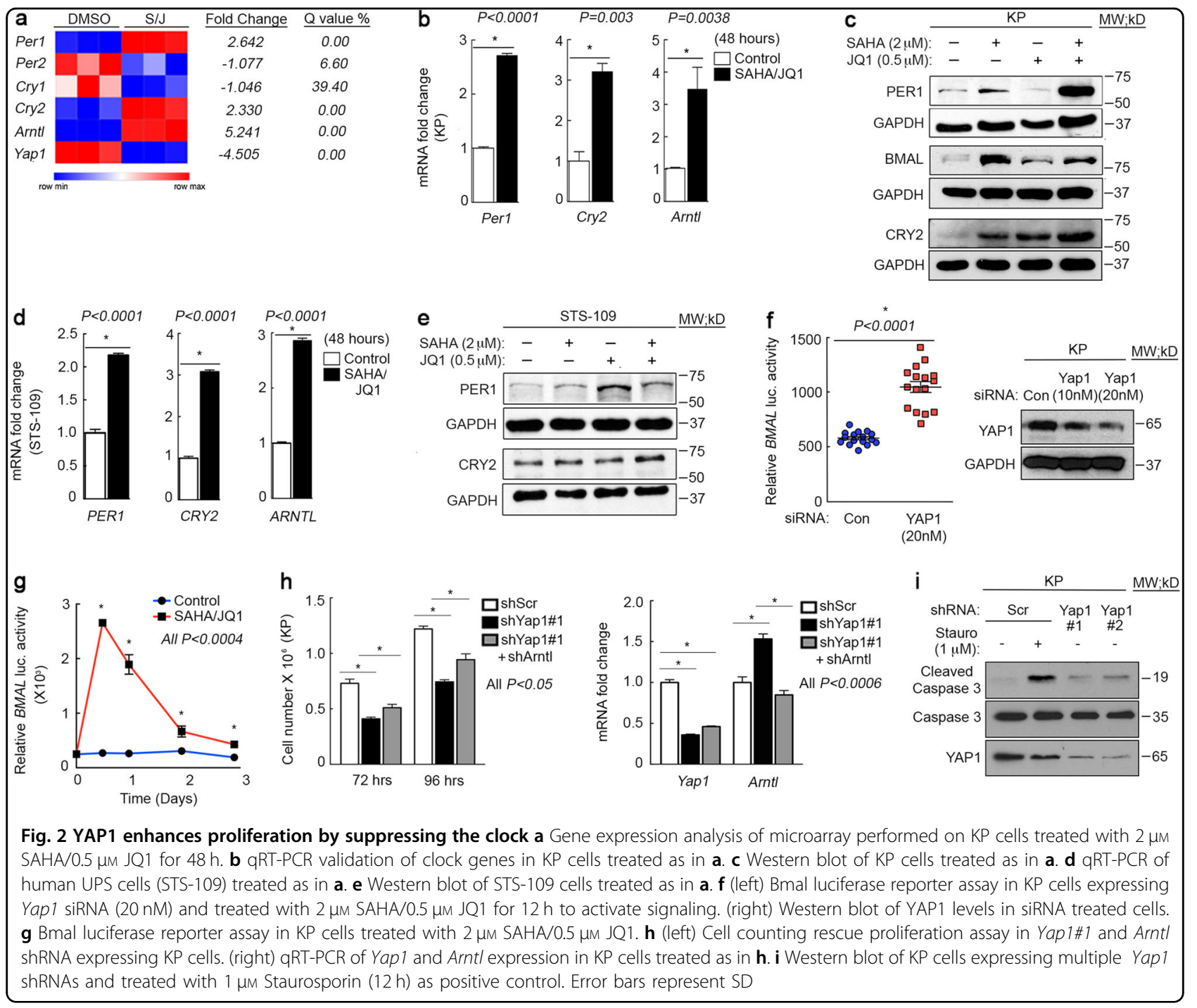

significantly rescued by Arntl inhibition (Fig. 3i). These data indicate that Rela-mediated suppression of the clock enhances proliferation.

YAP1/NF-KB loss initiates a clock-mediated UPR response

Recent studies have established a link between the UPR and the circadian clock $^{29,54}$. SAHA/JQ1 treatment upregulates multiple UPR target genes based on microarray analyses of KP cells (Fig. 4a). We validated induction of PERK and ATF6 pathways; two of three UPR branches, in SAHA/JQ1-treated cells (Fig. 4b, left) and cells expressing multiple independent Yap1 shRNAs (Fig. 4b, right). The main target of the third UPR branch, IRE1, is Xbp1. We did not observe $X b p 1$ upregulation or splicing in treated cells (Supplementary Fig. S4a). The well-studied PERK and ATF6 targets, Txnip and Ddit3/(Chop), were dramatically upregulated in SAHA/JQ1-treated KP cells (Fig. 4a). These targets are elevated in skeletal muscle but suppressed in fibrosarcomas and $\mathrm{UPS}^{50}$, suggesting that the UPR antagonizes sarcomagenesis in muscle-derived tumors (Fig. 4c, d). Consistent with these observations, TXNIP and CHOP protein expression increased in SAHA/JQ1-treated KP and HT-1080 cells (Fig. 4e-g) and KP cells expressing Yap1-specific shRNAs (Fig. 4h). To confirm upregulation of UPR in muscle, we evaluated Txnip and Ddit3 in C2C12 myoblasts and observed increased expression in differentiated cells (Fig. 4i). To determine whether NF- $\mathrm{kB}$ mediates Txnip and Ddit3 expression downstream of Yap1 we treated KP cells with BAY 11-7085 or Rela shRNAs and observed increases in Txnip and Ddit3 expression (Fig. 4j, k). Next, we performed a rescue experiment to determine whether Yap1mediated suppression of the circadian clock controls expression of Txnip and Ddit3. We treated KP cells with SAHA/JQ1 and Per1 shRNA alone or in combination. Interestingly, Txnip induction is dependent on clock 


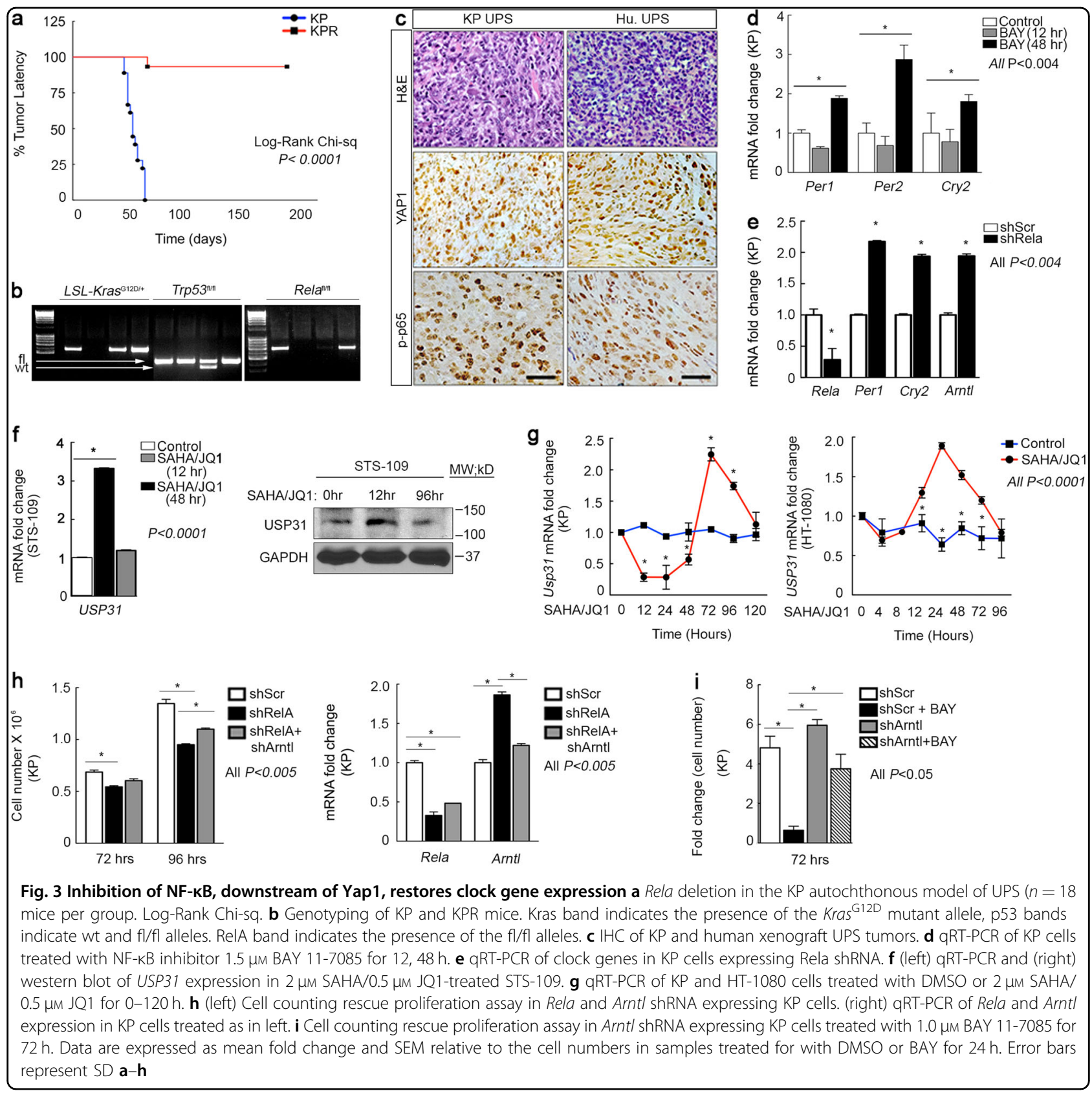

activity, whereas Ddit3 expression is not (Fig. 4l). Importantly, this observation agrees with our evaluation of Txnip and Ddit3 levels in differentiating C2C12 cells, wherein Txnip oscillates during a 6 day differentiation time course but Ddit3, simply increases at each time point (Fig. 4i). To ascertain the functional role of key UPR targets in SAHA/JQ1-induced differentiation we silenced Txnip and Ddit3 with specific shRNAs. We observed that loss of Txnip increases the expression of Ddit3 and the converse is true as well (Supplementary Fig. S4b). Therefore, we silenced both Txnip and Ddit3 in SAHA/ JQ1-treated cells to prevent compensation. Loss of Txnip and Ddit3 in SAHA/JQ-treated cells alters the normally cytostatic effects of these inhibitors and results in cell death, suggesting that UPR supports survival in differentiating muscle and sarcoma cells (Fig. $4 \mathrm{~m}, \mathrm{n}$ ). Thus, we conclude that the Yap1/NF- $\mathrm{kB}$ axis represses the PERK and ATF6 arms of the UPR, which are associated with clock activity and survival during differentiation ${ }^{29,54-56}$.

\section{SAHA/JQ1 treatment promotes oscillation of UPR target genes}

To explore the link between the UPR and circadian oscillation we characterized TXNIP and DDIT3 in both 


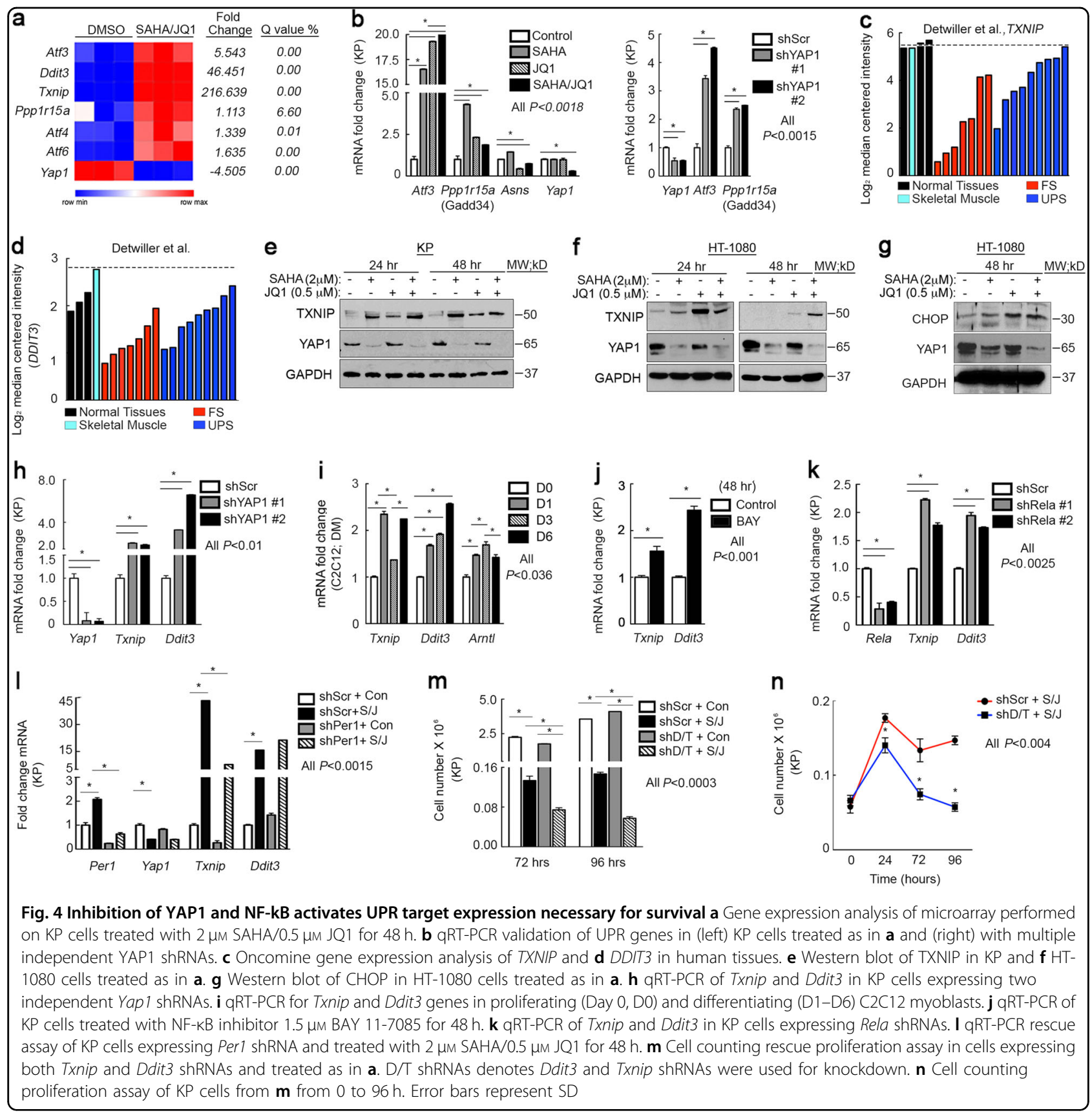

SAHA/JQ1-treated UPS and fibrosarcoma cells. TXNIP mRNA expression exhibited a dramatic circadian-like oscillation pattern with high amplitude changes in KP and HT-1080 cells over $0-120 \mathrm{~h}$ of treatment, whereas the amplitude of DDIT3 oscillations were more limited (Fig. 5a, b, Supplementary Fig. S5a and S5b). Oscillation was only observed in drug combination-treated cells, whereas individual drugs had minimal effects. Interestingly, YAP1 levels did not oscillate above 1 under these conditions, but instead decreased over time (Supplementary Fig. S5b). To test UPR engagement, in response to
SAHA/JQ1, we treated KP cells expressing ATF4-GFP and ATF6-GFP reporters for $24 \mathrm{~h}$ and found substantial increases in GFP reporter activity (Fig. 5c, d). To demonstrate that YAP1 suppresses UPR in vivo we stained tumor sections from allografts expressing control (scr) and Rela shRNA as well as our KP, KPY, and SAHA/JQ1-treated KP tumors for the UPR target Gadd34 and found that it was upregulated in Yap1/NF-kB-deficient tumors (Fig. 5e, f). Next, we evaluated TXNIP and CHOP expression in human tissues and observed decreased nuclear staining in UPS relative to muscle (Supplementary Fig. S5c). 


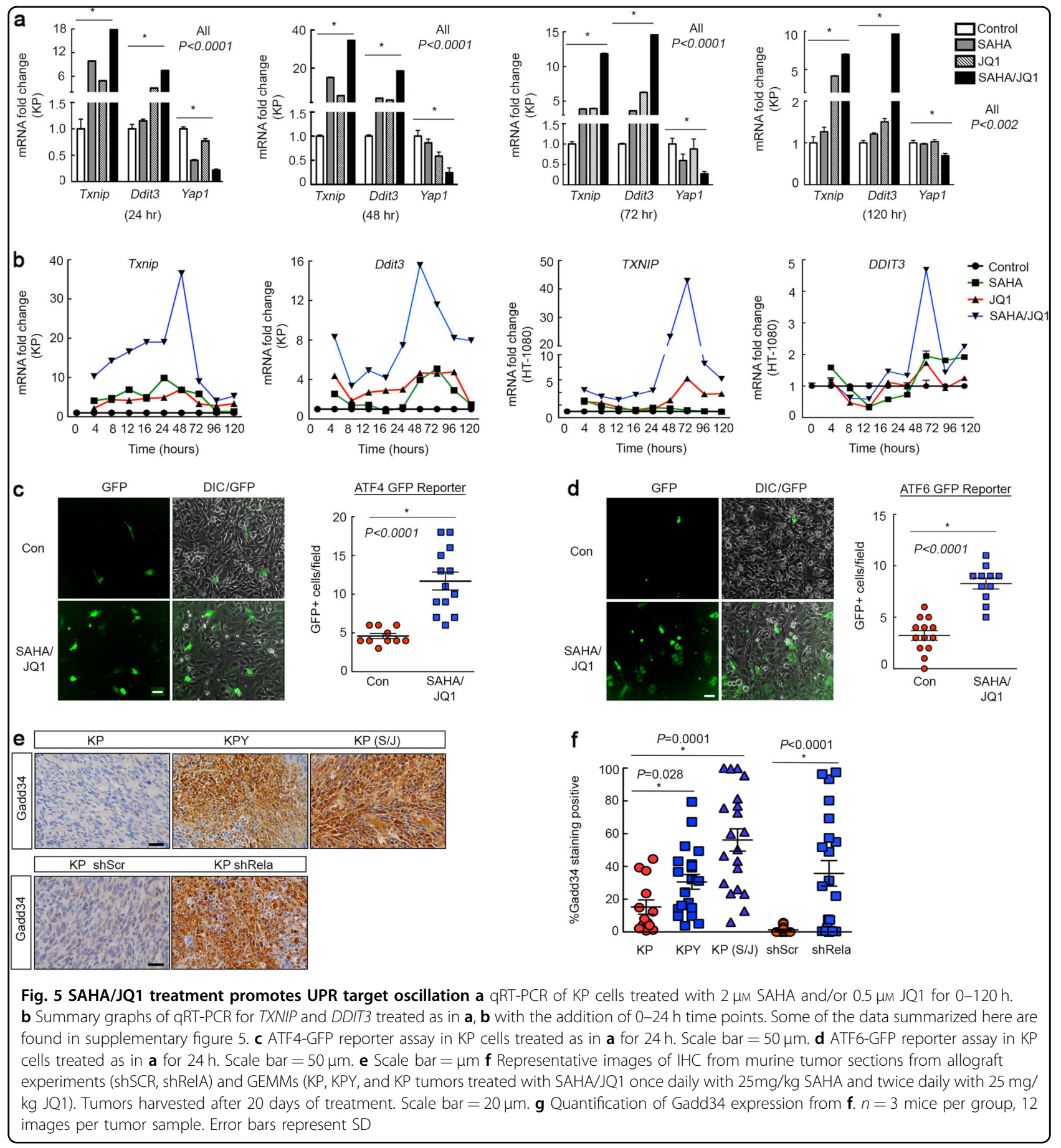

\section{Pharmacological and genetic inhibition of YAP1 alters sarcoma cell metabolism}

The primary known function of circadian circuitry is to regulate cellular metabolism ${ }^{20,57}$. Cancer cell metabolism is highly deregulated and favors the rapid, yet inefficient, energy production associated with glycolysis ${ }^{57}$. We hypothesized that reactivation of clock gene expression would alter the metabolic phenotype in sarcoma cells.
Therefore, we evaluated key metabolic genes in our microarray of SAHA/JQ1-treated KP cells and observed differential expression of metabolism hallmarks, such as Fatty acid synthase (Fasn) and Carnitine Palmitoyltransferase 1a and b (Cpt1a) (Cpt1b). CPT1A and CPT1B are associated with $\beta$-oxidation of fatty acids, a slower but more efficient metabolic process, found in non-malignant cells $^{58}$. CPT1B is the isoform generally associated with 


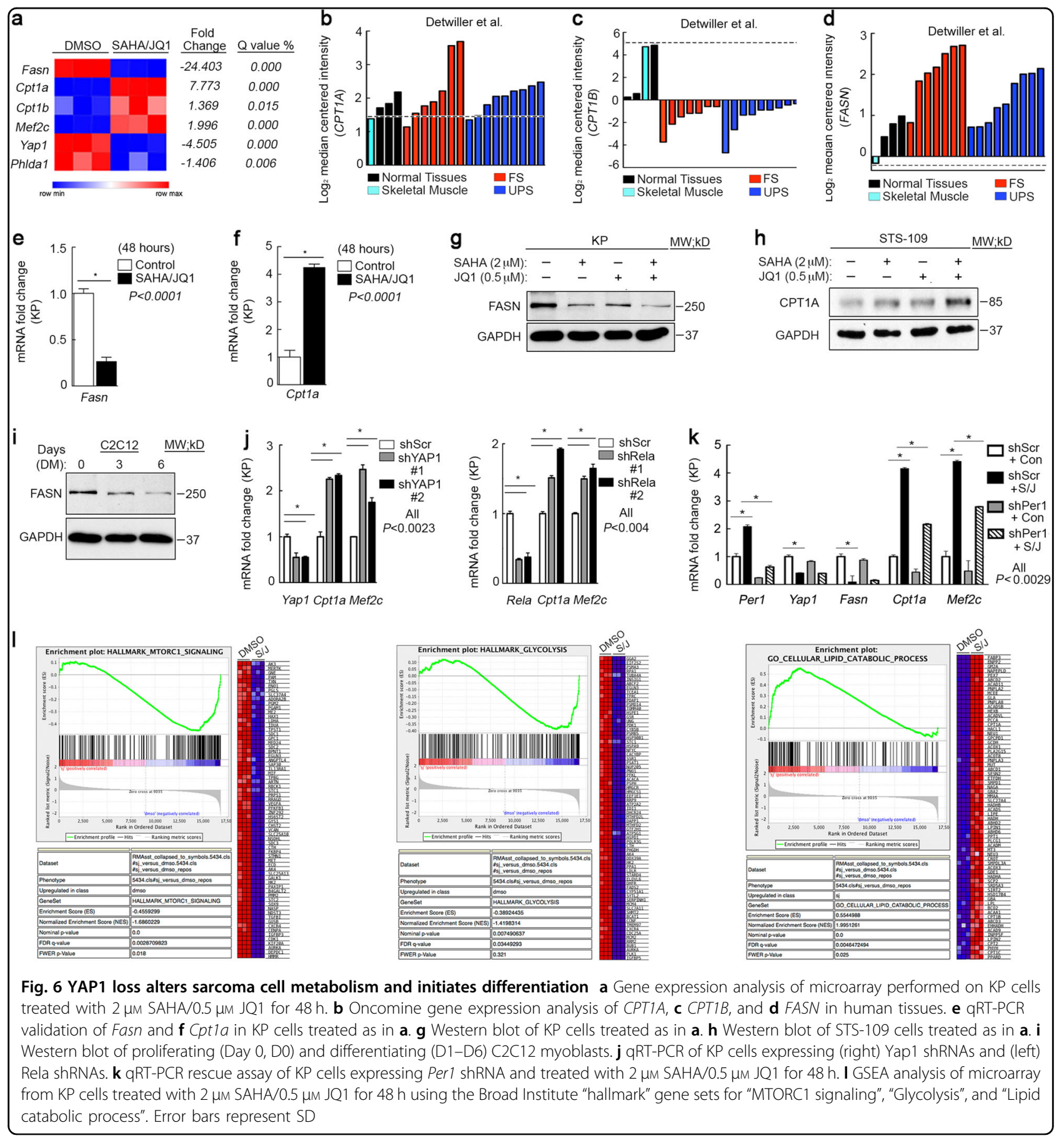

metabolism of differentiated muscle, whereas CPT1A can induce autophagy ${ }^{59}$. Fasn is associated with cancer metabolism and was downregulated dramatically in SAHA/JQ1-treated cells, whereas Cpt1a and Cpt1b and the muscle differentiation factor $M e f 2 c$ were elevated (Fig. 6a). In human UPS and fibrosarcoma CPT1A is modestly elevated or unchanged relative to normal tissues (Fig. 6b), whereas muscle-specific $C P T 1 B$ is lost and
FASN is increased (Fig. 6c, d). Importantly, SAHA/JQ1 treatment restored a more muscle-like metabolic program by decreasing Fasn and upregulating Cpt1a in KP and human UPS cells (Fig. 6e-h). Cpt1a induction may compensate for Cpt1b in this context (Fig. 6a). Consistent with our prediction that metabolic changes are associated with differentiation, Fasn is substantially decreased in differentiated $\mathrm{C} 2 \mathrm{C} 12$ cells (Fig. 6i). To determine whether 
these metabolic alterations were directly linked to the Yap1/NF-кB axis we inhibited Yap1 (Fig. 6j, left) or Rela (Fig. 6j, right) in KP cells with shRNA and observed increased Cpt1a and Mef2c expression, suggesting that YAP1 is necessary to suppress differentiation, potentially via $\beta$-oxidation of fatty acids or autophagy mediated by the CPT1 enzymes. Next, we performed a rescue experiment to determine whether YAP1-mediated suppression of the circadian clock controls metabolism and differentiation. We treated KP cells with SAHA/JQ1 and Per1 shRNA alone or in combination. We observed an increase in Cpt1a and Mef2c and loss of Yap1 under SAHA/JQ1 treatment as predicted, and whereas Per1 loss under these conditions prevented induction of Cpt1a and Mef2c (Fig. 6k). We observed the same trends in KP cells treated with SAHA/JQ1 and Arntl-specific shRNA (Supplementary Fig. S6a). To further characterize the metabolic status of SAHA/JQ1-treated KP cells we performed GSEA of the DMSO vs. SAHA/JQ1 microarray and observed that "MTORC1 signaling" and "Glycolysis" are downregulated in treated cells (Fig. 6l), consistent with the observations of other groups ${ }^{60,61}$, whereas "lipid catabolic processes" are upregulated. Together, these data show that the Yap1/ $\mathrm{NF}-\mathrm{kB}$ axis promotes cancer-associated metabolism and that inhibition of this pathway allows expression of muscle markers and muscle-associated metabolism.

YAP1, but not NF-KB, suppresses autophagy in UPS cells

In addition to its established role in fatty-acid oxidation, CPT1A is also associated with upregulation of autophagy $^{59}$. To determine which of these two processes occurs during SAHA/JQ1-mediated differentiation we performed GCMS evaluating oxidation of $75 \mu \mathrm{M}\left[\mathrm{U}_{-}{ }^{13} \mathrm{C}_{16}\right]$ palmitic acid and $75 \mu \mathrm{M}\left[\mathrm{U}_{-}{ }^{13} \mathrm{C}_{18}\right]$ oleic acid via enrichment of TCA cycle intermediates and observed a reproducible decrease in $\beta$-oxidation of fatty acids in SAHA/JQ1treated cells (Fig. 7a). These data suggest that increased expression of CPT1A may enhance oxidation of endogenous rather than exogenous lipids. Therefore, we investigated the ability of this drug combination to induce autophagy. Autophagy is directly associated with muscle function and maintenance of muscle mass ${ }^{32,34}$. The DMSO vs. SAHA/JQ1 microarray revealed that treatment upregulates many genes associated with autophagy including Atg13 and Atg14, which are specifically linked to muscle development and function (Fig. 7b). We also saw dramatic induction of the autophagy marker LC3 in KP cells treated with SAHA/JQ1 and Bafilomycin (BAF) (Fig. 7c, top). BAF interrupts autophagic flux by inhibiting maturation of autophagic vacuoles during the late stages of autophagy, forcing accumulation of the autophagosome-associated LC3-II. Consistently, shRNAmediated depletion of Yap1 enhanced LC3A/B expression in BAF-treated cells (Fig. 7c, bottom) and upregulated
Atg13 and Atg14 expression (Fig. 7d). Atg14 expression was also increased in KPY relative to KP tumors (Fig. 7e). Most significantly, we observed loss of p62 and dramatic accumulation of LC3B in vivo in KPY tumors and KP tumors treated with SAHA/JQ1 (Fig. 7f-h). SAHA/JQ1 treatment was initiated when KP tumors reached $100 \mathrm{~mm}^{3}$ according to the schedule found in (Supplementary Fig. S6b). Together, these findings link YAP1 to autophagy regulation in UPS. At last, we investigated whether YAP1-mediated suppression of autophagy was NF-kB- and clock-dependent. Rela inhibition had no effect on LC3A/B accumulation (Fig. 7i) or Atg13 and Atg14 levels (Supplementary Fig. S6c). Moreover, loss of Per1 in SAHA/JQ1-treated KP cells revealed that clock modulation has no effect on Atg13 and Atg14 (Fig. 7j) and that Atg13 does not oscillate at all during $\mathrm{C} 2 \mathrm{C} 12$ differentiation, whereas Atg14 oscillates extremely modestly (Supplementary Fig. S6d). These data indicate that autophagy is likely not required for muscle differentiation but is required for muscle maintenance and function as demonstrated by other groups ${ }^{34,62}$. At last, we investigated potential cross-talk between the UPR and autophagy. We treated KP cells expressing Txnip/Ddit3-specific shRNAs with SAHA/JQ1 and BAF. Txnip/Ddit3 inhibition had no effect on SAHA/JQ1-mediated autophagy (Supplementary Fig. S6e). We conclude from these observations that YAP1 suppresses autophagy independent of NF-kB signaling (Fig. 8a, b).

\section{Discussion}

Though well studied in epithelial tumors, the specific downstream effectors of YAP1 in sarcomas are still being elucidated. Characterization of these effectors is necessary for the development of effective biomarkers and targeted therapies to treat YAP1-dependent tumors including UPS. Previously, we showed that YAP1 promotes proliferation via persistent elevated NF- $\mathrm{KB}$ signaling ${ }^{7}$. The goal of this study was to elucidate the mechanisms by which YAP1 and NF- $\kappa B$ impact muscle de-differentiation and promote tumorigenesis. Here we show that YAP1-mediated NF- $k B$ signaling represses UPR and circadian clock activity, both of which are required for muscle differentiation ${ }^{17,56}$. We also show that YAP1 inhibits autophagy in an NF-kBindependent manner.

To our knowledge, this work provides the first direct link between the Hippo pathway and circadian clock activity. Using our autochthonous mouse models, we showed that Yap1-mediated NF- $\mathrm{kB}$ activity disrupts normal circadian oscillation by suppressing Per1, Per2, and Cry2 levels. Consistent with this observation, PER1, PER2, and CRY2 are downregulated in human UPS ${ }^{7}$. We also demonstrate that genetic or pharmacological inhibition of YAP1 enhances circadian clock activity and the oscillation of key targets including Txnip, which is a major effector of the UPR ${ }^{63,64}$. 


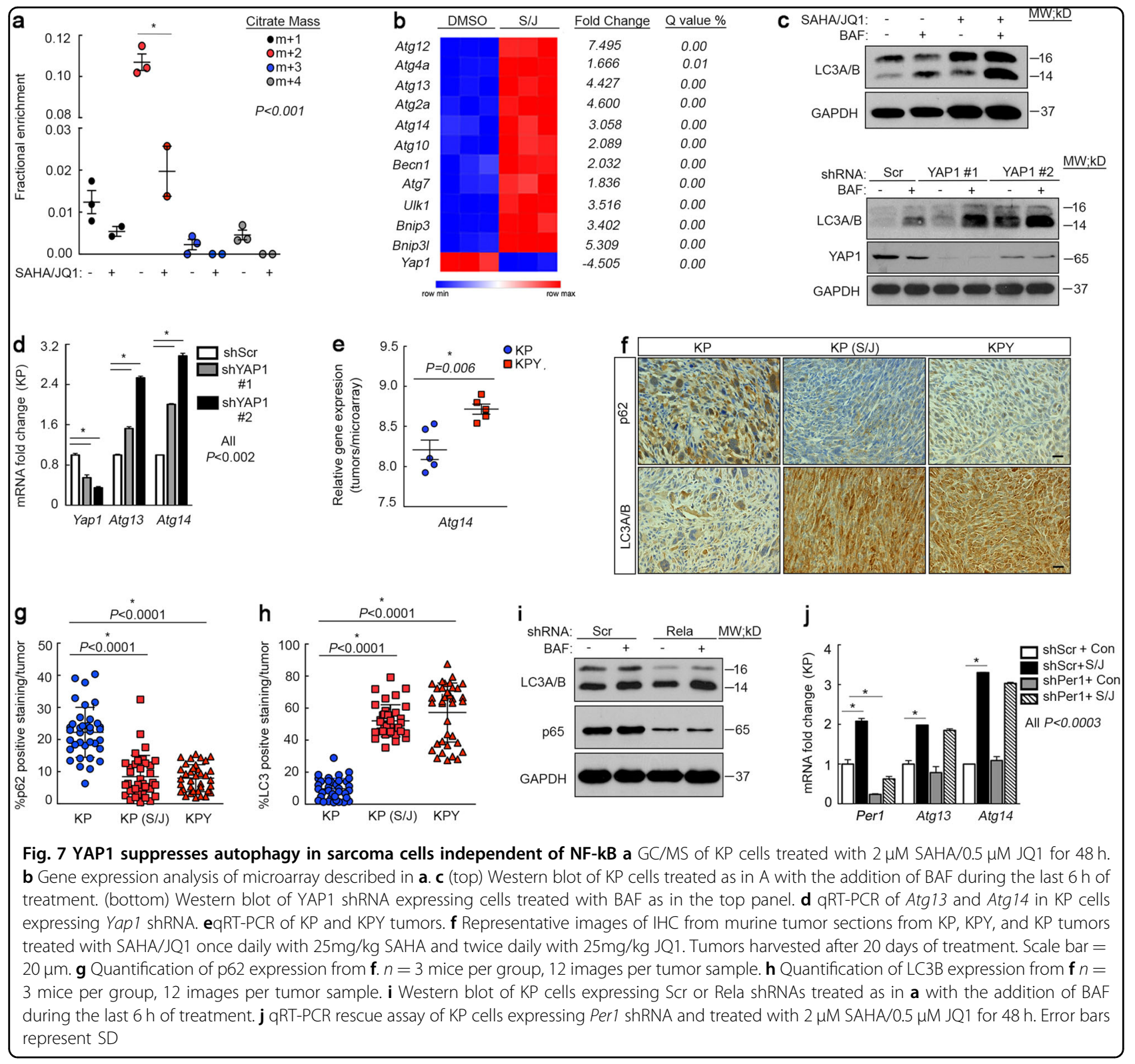

One critical purpose of the UPR and circadian clock is regulation of metabolic processes ${ }^{55}$. We found that Yap1mediated suppression of the UPR and clock support a shift in metabolism toward cancer cell-associated glycolysis and hyper-proliferation. Reactivation of the UPR and clock via SAHA/JQ1 correlate with decreased glycolysis while enhancing autophagy and lipid catabolism, thus promoting skeletal muscle differentiation.

Our work sheds light on several on-going areas of cancer research. First, our observation that the clock and the UPR are both activated in differentiating myoblasts, as well as SAHA/JQ1-treated sarcoma cells, indicates that the relationship between these two processes is contextdependent. In some tissues the UPR antagonizes clock gene expression $^{29}$, whereas in skeletal muscle and musclederived tumors both pathways are critical for differentiation and can be simultaneously upregulated ${ }^{17,56,65}$. This idea is consistent with several studies showing that UPR is necessary for muscle regeneration and maintenance of muscle mass ${ }^{55,56,65,66}$.

Clock function is also necessary for muscle generation and differentiation. In fact, MyoD, is a direct transcriptional target of the molecular clock $^{17,67,68}$. Moreover, clock-deficient mice suffer from muscle weakness, cachexia, and disrupted metabolism ${ }^{65}$. However, little is known about upstream signaling inputs that control clock gene expression and function. Now we appreciate that aberrant YAP1 stabilization impacts these processes in 


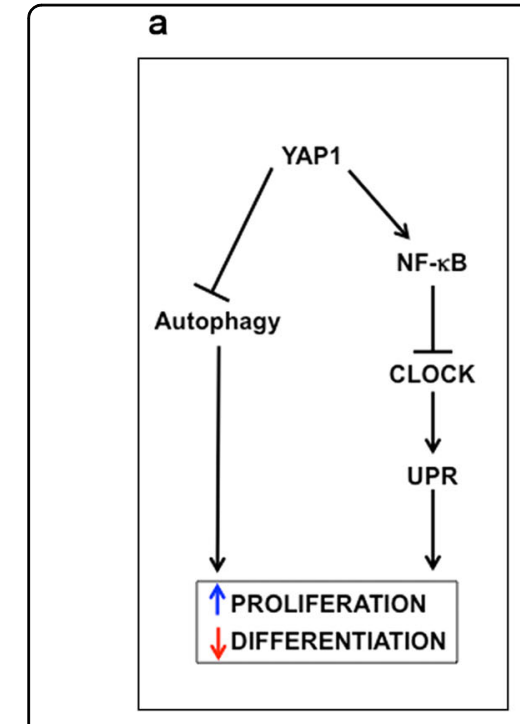

b

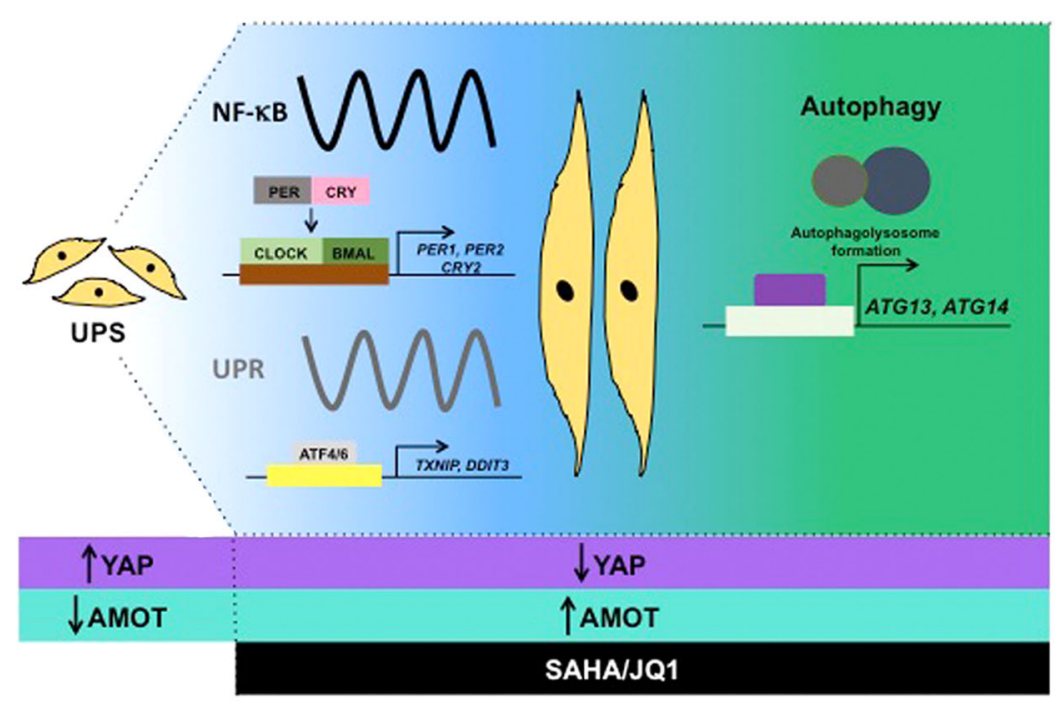

Fig. 8 Model of YAP1/NF-KB-mediated clock control Model of YAP1/NF-KB-mediated clock control of UPR and NF-KB-independent control of autophagy

muscle-derived sarcomas and potentially other contexts as well. In future studies, we will determine how YAP1 suppresses clock, UPR gene expression, and autophagy. YAP1 is generally considered a transcriptional activator. As such, our findings highlight novel roles for YAP1 in suppressing transcription. The targets repressed by YAP1 are particularly intriguing to us. Whereas, we might predict that YAP1 would inhibit expression of proapoptotic genes our work suggests that YAP1, via NF$\kappa B$, also represses pro-differentiation genes. This hypothesis is supported by data indicating that Yap1 inhibits muscle differentiation in $\mathrm{C} 2 \mathrm{C} 12$ myoblasts $^{10}$.

Finally we sought to determine the utility of epigenetic modulation in the treatment of muscle-derived sarcomas. We previously reported their impressive efficacy in the KP $\mathrm{GEMM}^{7}$. Here we validate our earlier finding that this strategy inhibits proliferation and enhances differentiation. We have identified key differentiation targets including clock, UPR, and autophagy genes as biomarkers of SAHA/JQ1 efficacy and suggest that this therapeutic strategy and these markers may offer clinical benefit to some sarcoma patients.

\section{Acknowledgements}

This work was funded by "Steps to Cure Sarcoma" (T.S.K.E.M.), the University of Pennsylvania Abramson Cancer Center (T.S.K.E.M.), the Penn Sarcoma Program (T.S.K.E.M.), The Sarcoma Foundation of America (T.S.K.E.M), The Slay Sarcoma Fund (T.S.K.E.M.), The University of Pennsylvania Cell and Molecular Biology training grant (T32 GM-07229) (A.R.R), NIH grants GM124749 (D.C.B.), The V foundation Scholar Award 3C59 8ABS3424 3BDA ((D.C.B), and the American Cancer Society Post-doctoral Fellowship 131203 PF 17 14701CCG
(J.M.P.). We thank John Tobias (Director, Molecular Profiling Facility, Univeristy of Pennsylvania) and Jennifer Shah for assistance with bioinformatics analyses.

\section{Author details}

${ }^{1}$ Abramson Family Cancer Research Institute, Department of Pathology \& Laboratory Medicine, Penn Sarcoma Program, University of Pennsylvania Perelman School of Medicine, Philadelphia, PA, USA. ${ }^{2}$ Department of Cancer Biology, University of Pennsylvania Perelman School of Medicine, Philadelphia, PA, USA. ${ }^{3}$ Department of Medicine, Harvard Medical School, Boston, MA 02115, USA. ${ }^{4}$ Department of Surgery and Institute of Medical Science, University of Toronto, Toronto, ON, Canada. ${ }^{5}$ Lunenfeld-Tanenbaum Research Institute, Sinai Health System, Toronto, ON, Canada. ${ }^{6}$ Department of Radiation Oncology, University of Pennsylvania Perelman School of Medicine, Philadelphia, PA, USA. ${ }^{7}$ Department of Radiology, University of Pennsylvania Perelman School of Medicine, Philadelphia, PA, USA. ${ }^{8}$ Department of Orthopedic Surgery, Penn Sarcoma Program, University of Pennsylvania Perelman School of Medicine, Philadelphia, PA, USA

\section{Author contributions}

A.R.R. and S.Y. designed the study, designed and performed most of the experiments, analyzed the data, and helped write the manuscript; G.M., S.E., G. C., S.C., Y.L., J.P., P.M.P., K.P., J.S.C., F.T., and N.M.L. performed experiments and data analysis; K.W. obtained human tumor samples from surgical patients; C.K., D.B., R.G., and J.Q. provided reagents, support, and experimental design assistance; T.S.K.E.M contributed to experimental design, study design, and data analysis and wrote the manuscript.

\section{Conflict of interest}

The authors declare that they have no conflict of interest.

\section{Publisher's note}

Springer Nature remains neutral with regard to jurisdictional claims in published maps and institutional affiliations.

Supplementary Information accompanies this paper at (https://doi.org/ 10.1038/s41419-018-1142-4). 
Received: 7 May 2018 Revised: 3 October 2018 Accepted: 10 October 2018 Published online: 31 October 2018

\section{References}

1. Pappo, A. S. et al. A phase 2 trial of R1507, a monoclonal antibody to the insulin-like growth factor-1 receptor (IGF-1R), in patients with recurrent or refractory rhabdomyosarcoma, osteosarcoma, synovial sarcoma, and other soft tissue sarcomas: results of a Sarcoma Alliance for Research Through Collaboration study. Cancer 120, 2448-2456 (2014).

2. Wagner, L. M. et al. Phase II study of cixutumumab in combination with temsirolimus in pediatric patients and young adults with recurrent or refractory sarcoma: a report from the Children's Oncology Group. Pediatr. Blood. Cancer 62, 440-444 (2015).

3. Taylor, B. S. et al. Advances in sarcoma genomics and new therapeutic targets. Nat. Rev. Cancer 11, 541-557 (2011).

4. Ballinger, M. L. et al. Monogenic and polygenic determinants of sarcoma risk: an international genetic study. Lancet Oncol. 17, 1261-1271 (2016).

5. Eisinger-Mathason, T. S. et al. Deregulation of the Hippo pathway in soft-tissue sarcoma promotes FOXM1 expression and tumorigenesis. Proc. Natl. Acad. Sci. USA 112, E3402-3411 (2015).

6. Mizuno, T. et al. YAP induces malignant mesothelioma cell proliferation by upregulating transcription of cell cycle-promoting genes. Oncogene 31, 5117-5122 (2012).

7. Ye, S. et al. YAP1-mediated suppression of USP31 enhances NF-kappaB activity to promote sarcomagenesis. Cancer Res. 78, 2705-2720 (2018).

8. Ye, S. \& Eisinger-Mathason, T. S. Targeting the Hippo pathway: clinical implications and therapeutics. Pharmacol. Res. 103, 270-278 (2016).

9. Rubin, B. P. et al. Evidence for an unanticipated relationship between undifferentiated pleomorphic sarcoma and embryonal rhabdomyosarcoma. Cancer Cell 19, 177-191 (2011).

10. Watt, K. I. et al. Yap is a novel regulator of C2C12 myogenesis. Biochem. Biophys. Res. Commun. 393, 619-624 (2010).

11. Tubaro, C., Arcuri, C., Giambanco, I. \& Donato, R. S100B protein in myoblasts modulates myogenic differentiation via NF-kappaB-dependent inhibition of MyoD expression. J. Cell Physiol. 223, 270-282 (2010).

12. Bakkar, N. et al. IKK/NF-kappaB regulates skeletal myogenesis via a signaling switch to inhibit differentiation and promote mitochondrial biogenesis. J. Cell Biol. 180, 787-802 (2008).

13. Dogra, C., Changotra, H., Mohan, S. \& Kumar, A. Tumor necrosis factor-like weak inducer of apoptosis inhibits skeletal myogenesis through sustained activation of nuclear factor-kappaB and degradation of MyoD protein. J. Biol. Chem. 281, 10327-10336 (2006).

14. Ardite, E., Barbera, J. A., Roca, J. \& Fernandez-Checa, J. C. Glutathione depletion impairs myogenic differentiation of murine skeletal muscle C2C12 cells through sustained NF-kappaB activation. Am. J. Pathol. 165, 719-728 (2004).

15. Zambrano, S., De Toma, I., Piffer, A., Bianchi, M. E. \& Agresti, A. NF-kappaB oscillationstranslate into functionally related patterns of gene expression. Elife 5, e09100 (2016).

16. Wang, X., Yu, W. \& Zheng, L. The dynamics of NF-kappaB pathway regulated by circadian clock. Math. Biosci. 260, 47-53 (2015).

17. Andrews, J. L. et al. CLOCK and BMAL1 regulate MyoD and are necessary for maintenance of skeletal muscle phenotype and function. Proc. Natl. Acad. Sci. USA 107, 19090-19095 (2010).

18. Zhang, X., Dube, T. J. \& Esser, K. A. Working around the clock: circadian rhythms and skeletal muscle. J. Appl. Physiol. 107, 1647-1654 (2009).

19. McCarthy, J. J. et al. Identification of the circadian transcriptome in adult mouse skeletal muscle. Physiol. Genom. 31, 86-95 (2007).

20. Bass, J. \& Takahashi, J. S. Circadian integration of metabolism and energetics. Science 330, 1349-1354 (2010).

21. Huang, G., Zhang, F., Ye, Q. \& Wang, H. The circadian clock regulates autophagy directly through the nuclear hormone receptor Nr1d1/Rev-erbalpha and indirectly via Cebpb/(C/ebpbeta) in zebrafish. Autophagy 12, 1292-1309 (2016).

22. Bell-Pedersen, D. et al. Circadian rhythms from multiple oscillators: lessons from diverse organisms. Nat. Rev. Genet. 6, 544-556 (2005).

23. Lowrey, P. L. \& Takahashi, J. S. Mammalian circadian biology: elucidating genome-wide levels of temporal organization. Annu. Rev. Genomics Hum. Genet. 5, 407-441 (2004)
24. Ripperger, J. A. \& Schibler, U. Rhythmic CLOCK-BMAL1 binding to multiple Ebox motifs drives circadian Dbp transcription and chromatin transitions. Nat. Genet. 38, 369-374 (2006).

25. The Circadian Clock Protein CRY2. Promotes MYC degradation. Cancer Discov. 7, OF16 (2017).

26. $\mathrm{Wu}, \mathrm{S}$., Fesler, A. \& Ju, J. Implications of circadian rhythm regulation by microRNAs in colorectal cancer. Cancer Transl. Med 2, 1-6 (2016).

27. Ha, N. H., Long, J., Cai, Q., Shu, X. O. \& Hunter, K. W. The circadian rhythm gene Arntl2 is a metastasis susceptibility gene for estrogen receptor-negative breast cancer. PLoS Genet. 12, e1006267 (2016).

28. Fu, L., Pelicano, H., Liu, J., Huang, P. \& Lee, C. The circadian gene Period2 plays an important role in tumor suppression and DNA damage response in vivo. Cell 111, 41-50 (2002)

29. $\mathrm{Bu}, \mathrm{Y}$. et al. A PERK-miR-211 axis suppresses circadian regulators and protein synthesis to promote cancer cell survival. Nat. Cell Biol. 20, 104-115 (2018).

30. Koyanagi, S. et al. CAMP-response element (CRE)-mediated transcription by activating transcription factor-4 (ATF4) is essential for circadian expression of the Period2 gene. J. Biol. Chem. 286, 32416-32423 (2011).

31. Igarashi, T. et al. Clock and ATF4 transcription system regulates drug resistance in human cancer cell lines. Oncogene 26, 4749-4760 (2007).

32. Fortini, $P$. et al. The fine tuning of metabolism, autophagy and differentiation during in vitro myogenesis. Cell Death Dis. 7, e2168 (2016).

33. Lee, W. S., Yoo, W. H. \& Chae, H. J. ER stress and autophagy. Curr. Mol. Med. 15 735-745 (2015).

34. Masiero, E. et al. Autophagy is required to maintain muscle mass. Cell Metab. 10, 507-515 (2009)

35. Wang, J. et al. Hepatitis C virus core protein activates autophagy through EIF2AK3 and ATF6 UPR pathway-mediated MAP1LC3B and ATG12 expression. Autophagy 10, 766-784 (2014).

36. Margariti, A. et al. XBP1 mRNA splicing triggers an autophagic response in endothelial cells through BECLIN-1 transcriptional activation. J. Biol. Chem. 288, 859-872 (2013).

37. Gade, P. et al. An IFN-gamma-stimulated ATF6-C/EBP-beta-signaling pathway critical for the expression of Death Associated Protein Kinase 1 and induction of autophagy. Proc. Natl. Acad. Sci. USA 109, 10316-10321 (2012).

38. Rzymski, T. et al. Regulation of autophagy by ATF4 in response to severe hypoxia. Oncogene 29, 4424-4435 (2010).

39. Song, Q. et al. YAP enhances autophagic flux to promote breast cancer cell survival in response to nutrient deprivation. PLOS ONE 10, e0120790 (2015).

40. $\mathrm{Wu}, \mathrm{H}$. et al. Integration of Hippo signalling and the unfolded protein response to restrain liver overgrowth and tumorigenesis. Nat. Commun. 6, 6239 (2015).

41. Eisinger-Mathason, T. S. et al. Hypoxia-dependent modification of collagen networks promotes sarcoma metastasis. Cancer Discov. 3, 1190-1205 (2013).

42. Subramanian, A. et al. Gene set enrichment analysis: a knowledge-based approach for interpreting genome-wide expression profiles. Proc. Natl. Acad. Sci. USA 102, 15545-15550 (2005).

43. Berezowska, S. \& Galván, J. A. Immunohistochemical detection of the autophagy markers LC3 and p62/SQSTM1 in formalin-fixed and paraffin-embedded tissue. Methods Mol. Biol. 1560, 189-194 (2017).

44. Ruifrok, A. C. \& Johnston, D. A. Quantification of histochemical staining by color deconvolution. Anal. Quant. Cytol. Histol. 23, 291-299 (2001).

45. Millard, P., Letisse, F., Sokol, S. \& Portais, J. C. IsoCor: correcting MS data in isotope labeling experiments. Bioinformatics 28, 1294-1296 (2012).

46. Mito, J. K. et al. Cross species genomic analysis identifies a mouse model as undifferentiated pleomorphic sarcoma/malignant fibrous histiocytoma. PLoS ONE 4, e8075 (2009).

47. Kirsch, D. G. et al. A spatially and temporally restricted mouse model of soft tissue sarcoma. Nat. Med. 13, 992-997 (2007).

48. Serrano, C. et al. RAS/MAPK pathway hyperactivation determines poor prognosis in undifferentiated pleomorphic sarcomas. Cancer 122, 99-107 (2016).

49. Kondratov, R. V., Kondratova, A. A., Gorbacheva, V. Y., Vykhovanets, O. V. \& Antoch, M. P. Early aging and age-related pathologies in mice deficient in BMAL1, the core componentof the circadian clock. Genes Dev. 20, 1868-1873 (2006).

50. Detwiller, K. Y. et al. Analysis of hypoxia-related gene expression in sarcomas and effect of hypoxia on RNA interference of vascular endothelial cell growth factor A. Cancer Res. 65, 5881-5889 (2005).

51. Cancer Genome Atlas Research Network. Electronic address: elizabeth demicco@sinaihealthsystem.ca1; Cancer Genome Atlas Research Network 
Comprehensive and integrated genomic characterization of adult soft tissue sarcomas. Cell 171, 950-965 e928 (2017).

52. Bai, $H$. et al. Expression of Yes-associated protein modulates Survivin expression in primary liver malignancies. Hum. Pathol. 43, 1376-1385 (2012).

53. Liu, T. et al. YAP1 up-regulation inhibits apoptosis of aortic dissection vascular smooth muscle cells. Eur. Rev. Med. Pharmacol. Sci. 21, 4632-4639 (2017).

54. Yuan, G. et al. Clock mediates liver senescence by controlling ER stress. Aging 9, 2647-2665 (2017).

55. Bohnert, K. R., McMillan, J. D. \& Kumar, A. Emerging roles of ER stress and unfolded protein response pathways in skeletal muscle health and disease. $J$. Cell Physiol. 233, 67-78 (2018).

56. Xiong, G. et al. The PERK arm of the unfolded protein response regulates satellite cell-mediated skeletal muscle regeneration. Elife 6, pii: e22871 (2017).

57. Altman, B. J. et al. MYC disrupts the circadian clock and metabolism in cancer cells. Cell Metab. 22, 1009-1019 (2015).

58. Carracedo, A., Cantley, L. C. \& Pandolfi, P. P. Cancer metabolism: fatty acid oxidation in the limelight. Nat. Rev. Cancer 13, 227-232 (2013).

59. Niso-Santano, M. et al. Unsaturated fatty acids induce non-canonical autophagy. EMBO J. 34, 1025-1041 (2015).

60. Hu, J. K. et al. An FAK-YAP-mTOR signaling axis regulates stem cell-based tissue renewal in mice. Cell Stem. Cell 21, 91-106 e106 (2017).
61. Park, Y. Y. et al. Yes-associated protein 1 and transcriptional coactivator with PDZ-binding motif activate the mammalian target of rapamycin complex 1 pathway by regulating amino acid transporters in hepatocellular carcinoma. Hepatology 63, 159-172 (2016).

62. Sandri, M., Coletto, L., Grumati, P. \& Bonaldo, P. Misregulation of autophagy and protein degradation systems in myopathies and muscular dystrophies. J. Cell Sci. 126, 5325-5333 (2013).

63. Oslowski, C. M. et al. Thioredoxin-interacting protein mediates ER stressinduced beta cell death through initiation of the inflammasome. Cell Metab. 16, 265-273 (2012).

64. Ma, Y., Brewer, J. W., Diehl, J. A. \& Hendershot, L. M. Two distinct stress signaling pathways converge upon the CHOP promoter during the mammalian unfolded protein response. J. Mol. Biol. 318, 1351-1365 (2002).

65. Bohnert, K. R. et al. Inhibition of ER stress and unfolding protein response pathways causes skeletal muscle wasting during cancer cachexia. FASEB J. $\mathbf{3 0}$ 3053-3068 (2016).

66. Nakanishi, K., Dohmae, N. \& Morishima, N. Endoplasmic reticulum stress increases myofiber formation in vitro. FASEB J. 21, 2994-3003 (2007).

67. Zhang, X. et al. A non-canonical E-box within the MyoD core enhancer is necessary for circadian expression in skeletal muscle. Nucleic Acids Res. 40 3419-3430 (2012).

68. Lefta, M., Wolff, G. \& Esser, K. A. Circadian rhythms, the molecular clock, and skeletal muscle. Curr. Top. Dev. Biol. 96, 231-271 (2011). 